\title{
Adaptive Modulation Control for Visible Light Communication Systems
}

\author{
Antonio Costanzo ${ }^{(1)}$, Member, IEEE, Valeria Loscri $\left.{ }^{(}\right)$, Senior Member, IEEE, \\ and Mauro Biagi ${ }^{\circledR}$, Senior Member, IEEE
}

\begin{abstract}
Visible light communication (VLC) builds on the dual use of lightening infrastructure for communication. Even though the advantages of VLC are well known, as emerging communication paradigm, some open issues still need to be addressed in order to rely on it as a robust communication system. First of all, external interference as an extremely varying signal impacting on the reliability of the VLC system needs to be analyzed. In this article, we propose a system where the link conditions (in terms of signal-to-noise-ratio (SNR)) drive the modulation scheme and this procedure is managed through the use of an uplink/channel, to assure a feedback path. The receiver is in charge of choosing the modulation scheme matching the requirement in terms of error rate on the basis of the measured SNR after noise mitigation. The feasibility of the system and its effectiveness are evaluated by designing and implementing a complete bi-directional system. In particular, an uplink channel sending the information regarding the specific selected modulation technique has been implemented and the whole system is based on a fine synchronization approach in order to "track" in real time the most suitable modulation scheme. Experimental results show the effectiveness of a bi-directional system in order to implement an adaptive VLC system able to follow the environmental changes (in terms of interference and noise).
\end{abstract}

Index Terms-Adaptive modulation, noise mitigation, SNR, visible light communication.

\section{INTRODUCTION}

$\mathbf{V}$ ISIBLE Light Communication (VLC) [1] paradigm envisages the use of Light Emitting Diodes (LED) for data communication. In other words, VLC could be considered as an extension of optical wireless communication (OWC) to lampshade lights. In recent years, LED lights have become more and more convenient in comparison to other lighting systems, like fluorescent sources; the ratio between LED electrical power consumption and brightness considerably decreased in last years,

Manuscript received June 19, 2020; revised October 9, 2020 and December 11, 2020; accepted January 28, 2021. Date of publication February 2, 2021; date of current version May 2, 2021. This work was supported by a grant from CPER/FEDER DATA project and by the Region Hauts de France, project LUMICAR, in the framework of "STIMuLE: Support for Interdisciplinary, Multi-establishment and Exploratory Research" under Grant 19004007. (Corresponding author: Antonio Costanzo.)

Antonio Costanzo and Valeria Loscri are with the Inria LilleNord Europe, 59650 Villeneuve-d'Ascq, France (e-mail: antonio. costanzo@inria.fr; valeria.loscri@inria.fr).

Mauro Biagi is with the Deptartment of Information Electrical and Telecommunication (DIET) Engineering, Sapienza University of Rome, 00184 Rome, Italy (e-mail: mauro.biagi@uniroma1.it).

Color versions of one or more figures in this article are available at https: //doi.org/10.1109/JLT.2021.3056177.

Digital Object Identifier 10.1109/JLT.2021.3056177 as well as the price of the radiating elements. Long lifetime, physical robustness, small size, and fast switching are the main reasons why LED lamps are widely replacing other illumination devices. An infrastructure that can be simultaneously employed for illumination and data communication, allows a huge long term energy saving. Furthermore, VLC presents several other advantages in comparison to radio frequency (RF) and microwave communication systems. First of all, VLC could be exploited in all those places where RF solutions represent a dangerous source of interference in critical environments (e.g. hospitals, aircrafts, mines and petrochemical plants). VLC could be used for short distance underwater communication [2], [3], because the absorption coefficient of water at optical frequencies (especially in the blue range), is several times lower than the one shown at lower frequencies of the electromagnetic spectrum. VLC paradigm has also been considered to obtain affordable indoor positioning operations [4], [5], and portend future vehicle to vehicle communication [6]. Since frequency spectrum has become significantly crowded in latter years, with a possible saturation in the next decade, the integration of VLC system could significantly mitigate this problem.

However, even if this VLC paradigm involves indisputable advantages, improving the quality of the optical signal to achieve higher performance, currently represents a main issue to obtain an effective applicability in real scenarios.

\section{A. State of Art}

One of the main challenges is to adapt existing signal processing techniques, available for classical wireless communication, to the signal diffused by LED lamps. Indeed, data transmission in VLC systems is usually performed by intensity modulation and direct detection. According to this technique, information can be transmitted and received only using real and positive signals. Conventional modulation schemes, like on-off keying (OOK), pulse-amplitude modulation (PAM), pulse position modulation (PPM), frequency shift keying (FSK) and Optical orthogonal frequency-division multiplexing (OFDM) modulation [8] have been properly modified for allowing the processing of VLC signals. Regardless of modulation scheme, the main issue is represented by the high level of interference caused by external light sources and the extremely variability of this interference in a real scenario. Recently, it has been also experimentally demonstrated that other source of interference can seriously impact on the performance of a VLC system [18]. In particular, 
sunlight can completely waste the quality of received optical signals, especially in outdoor environments. Other noise sources are represented by thermal and shot noise. Thermal noise, caused by random motion of electrons or charges, is mainly generated during the amplifying stage. Shot noise is related to the total amount of light incident on the photo-detector. Typically, in conventional methods, high-pass filters are adapted to block direct current (DC) signals affected by noise [9]. However, since optical signals are modulated as square waves, filtering process is not sufficient to achieve a clean output. In [10] spectral line interference is theoretically analysed and approximately mitigated by adopting a set of improved waveform samples; in [11] noise effects on synchronization are reduced using a learning technique based on multi-armed bandit; an effective noise mitigation approach, based on the noise listening and successive noise mitigation to limit the effects of external lights on Symbol Error Rate (SER) has been introduced in [12].

\section{B. Goals and Organization of the Work}

Among the first contributions in terms of software defined open platforms for Visible Light Communications are [19] and [20], where the authors demonstrate the feasibility of the communication based on visible light by the means of a software defined approach and based on the use of low-cost commercial devices.

One of the first and most advanced software defined open platforms for Visible Light Communication is OpenVLC [21], where the authors highlight the importance and difficulty of synchronization between a receiver and a transmitter in a bidirectional VLC system. The approach considered by the authors to avoid mistiming issue consists in oversampling. In [22] the authors implement a unidirectional VLC platform and demonstrate the OFDM implementation.

To the best of our knowledge there are not real platforms implementing a robust adaptive communication system based on visible light, integrating a noise mitigation approach for making the system suitable to adapt to the most efficient, in terms of energy and spectral efficiency, modulation scheme and implementing a robust synchronization scheme between the transmitter and the receiver in order to make the system able to react in real time to the dynamic of the external changes.

In most of previous works, considering adaptive mechanisms, a unidirectional data stream is considered. Hence, for fully taking advantage of adaptive schemes in real implementation, it is mandatory to consider an uplink channel. Indeed, when the external conditions vary, the system can report the most suitable modulation to be selected by the means of this channel used as a feedback link. Based on that, in this work we design and implement a VLC system in which we consider an uplink channel between the receiver $(\mathrm{Rx})$ and the transmitter $(\mathrm{Tx})$ nodes in order to effectively adapt the parameters of the system based on the surrounding changes. Of course, this kind of implementation implies new challenges, above all in terms of synchronization between the data flow transmission between the Tx and Rx and the corresponding uplink/feedback channel.

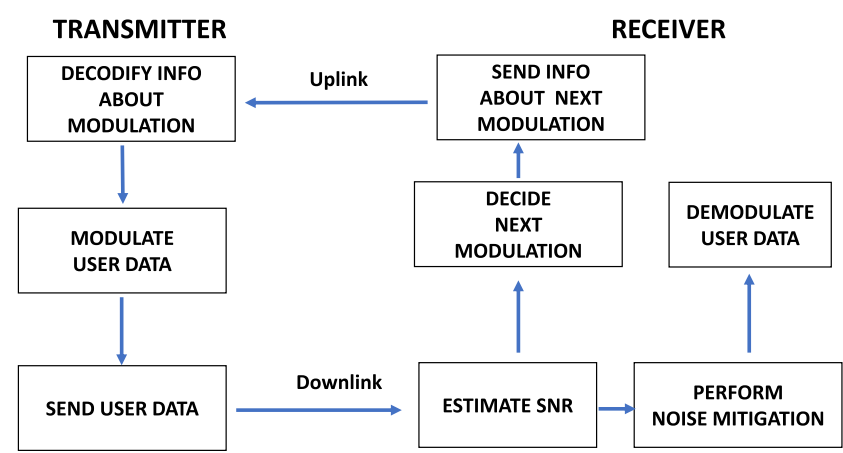

Fig. 1. Adaptive modulation control system for VLC: main operations.

The main contributions of this work can be summarized as follows:

- We propose a novel bidirectional VLC system capable to select the most suitable modulation scheme according to the reliability level and based on the Signal to Noise Ratio (SNR). The implementation utilizes a two-way VLC link since we do not consider here illumination constraints. Differently, in the case of ceiling-to-floor communication it is important to pay attention for the uplink channel due to light coming from floor-to-ceiling.

- We apply a noise mitigation approach as defined in [12], with the main difference that we integrate this approach to work in real-time. In practice, the variations of external conditions directly impact on the performance of the system evaluated in terms of SNR and thanks to a fine-coarse synchronized uplink channel, the communication system is able to adapt with the most appropriate modulation scheme.

- By the means of a software defined approach [16], [17], we design and implement the whole communication protocol, integrating the noise mitigation, the synchronization approach and we demonstrate not only the feasibility of such a type of systems but also the effectiveness in different real scenarios.

The paper is organized as follows. After introducing the system model in section II, we describe the different procedures including synchronization, channel estimation, modulation control and detection in section III. We give the details of the system implementation in section IV. The performance of the proposed system are reported in section V, while in section VI we conclude the work.

\section{SySTEM MODEL}

We assume to have a downlink channel where transmission and detection take place and an uplink channel, from receiver to transmitter, providing a feedback about the modulation scheme to employ in the next frame. The main actions performed by the system are described in Fig. 1.

\section{A. Downlink Channel}

Transmitted signal is allowed to have a maximum power level equating $P^{\max }$. The modulation schemes we consider in this work are both non-orthogonal and orthogonal, namely 2-, 4-, 8-, 


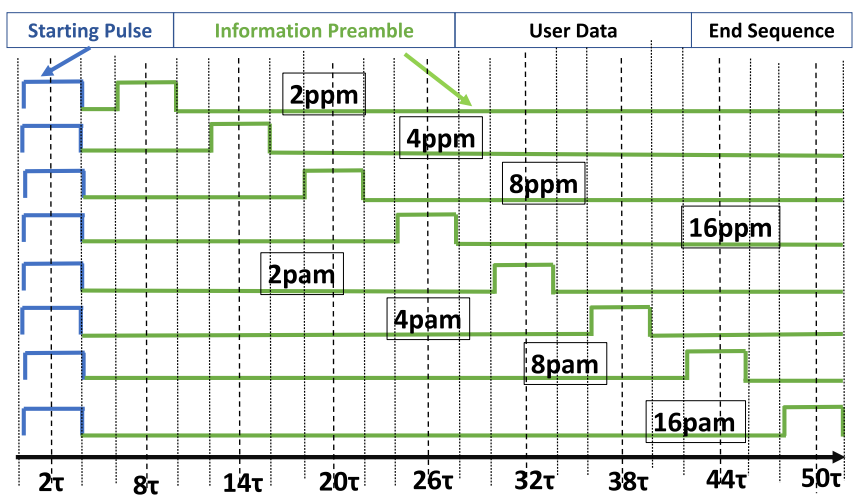

Fig. 2. Downlink Communication: Starting pulse and identification preamble for all available modulations.

16- PAM and 2-, 4-, 8-, 16- PPM. As known from communication theory, the former let increase rate at the expense of error rate while the latter let achieve high reliability at the expense of transmission rate with increasing of the modulation index. In the case of PAM, the transmitted signal is

$$
s_{a}(t)=A_{\ell} x(t)
$$

with $A_{\ell}$ belonging to the $\mathrm{M}$-ary set of amplitudes characterizing the PAM alphabet, $x(t)=A_{0} \Pi\left(\frac{t-T_{p}}{\tau}\right)$ represents the rectangular transmitting pulse, delayed by $T_{p}$ and with duration $\tau$, driving the LED before applying the modulation. $A_{0}=12 \mathrm{~V}$ is the constant amplitude of the pulse driving the LED in our transmitting circuit. The PPM signalling is represented by the following relationship

$$
s_{d}(t)=x\left(t-\Delta_{\ell} T_{p}\right)
$$

where $\Delta_{\ell}$ describes the delay ranging from 0 to $\mathrm{M}-1$ as multiple integer of $T_{p}$ elementary delay equating, at least, the pulse length in order to grant orthogonality among PPM symbols. A fair comparison among those two modulations is not trivial since while PPM assures to have an illumination equating $P^{\max }$ in a PPM symbol, PAM is not able and a proper amplitude setting must be carried out. For sake of fairness we consider that each PPM symbol presents an intensity equating $P^{\max }$ so the energy among a PPM symbol is $E_{P P M}=P^{\max } / M T_{p}$. For PAM we consider to have the same energy (average) so the amplitudes are in the interval $\left\{0, \ldots, 2 E_{P P M} T_{p}\right\}$. In other words we consider to have the same illumination level for PPM and PAM.

In order to maintain a high level of flexibility, and avoid as much as possible overhead and additive signal processing, data frame involved in the downlink optical communication includes only a starting pulse, an information pulse (which identifies the type of modulation), user data and an end sequence, as shown in Fig. 2.

The starting pulse with duration $T_{\text {start }}$, lasts 4 times the pulse length of the PPM modulation one and it is used by the receiver for synchronization issue and identifying the beginning of the frame. In the description, we consider the notation $A_{0} \Pi\left(\frac{t-a}{b}\right)$, which represents the rectangular function centered at $a$, with duration $b$ and amplitude $A_{0}$. Since we consider the beginning of the starting pulse as zero time reference, $a=2 \tau$ and
$b=T_{\text {start }}=4 \tau$. Considering the signal driving the LED before applying the modulation, $A_{0}$ is the constant amplitude of the pulse driving the LED. In our transmitting circuit, $A_{0}=12 \mathrm{~V}$. The starting pulse is identical for each modulation scheme adopted in the system, namely:

$$
s_{\text {start }}(t)=A_{0} \Pi\left(\frac{t-2 \tau}{4 \tau}\right)
$$

The information pulse, with duration $T_{s}$, codifies, through a PPM signal, the type of modulation the receiver has to set for data recovering. We maintained, for the information pulse, the same width of the starting pulse, so $T_{s}=T_{\text {start }}=4 \tau$.

In order to avoid synchronization errors, which could lead to misunderstand the correct modulation, guard intervals are placed between the starting pulse and the information preamble, between each possible pulse position and between the information preamble and codified user data. The width of guard intervals is twice the pulse adopted by PPM modulation $\left(T_{g}=2 \tau\right)$. We chose guard intervals at least twice the pulse, in order to easily avoid ambiguity due to rising and falling edges, clock drifts and other phenomena. Indeed, an error in retrieving the correct position of the pulse representing information preamble, would completely corrupt the whole content of user data. We chose the above values for starting and synchronization pulses, for avoiding any possible ambiguity with user data. In our case, a starting pulse equal to $4 \tau$ is long enough to avoid false trigger caused by background light and other external optical sources.

Furthermore, being $M_{P P M}$ the order of PPM modulation used by the transmitter $(2,4,8$ or 16$)$, and following the same time reference used above, the associated synchronization pulse is:

$$
s_{M_{P P M}}(t)=A_{0} \Pi\left(\frac{t-a_{P P M}}{b_{P P M}}\right)
$$

where $b_{P P M}=4 \tau$ and, $a_{P P M}$, referring to Fig. 2, is

$$
a_{P P M}=6 \tau+\log _{2}\left(M_{P P M}\right) T_{g}+\left(\log _{2}\left(M_{P P M}\right)-1\right) T_{s}
$$

According to the time representation in Fig. 2, the first term in Eq. (5) refers to the first possible pulse position (the one indicating 2PPM), which, in our implementation, is equal to $T_{\text {start }}+T_{g}=6 \tau$. For further modulation indexes, the pulses are sequentially delayed by an additive $T_{g}$ (second term in Eq. (5)) plus a $T_{s}$ (last term).

In order to avoid complex synchronization schemes, PAM modulations are indicated with the same mechanism. A further delay $a_{P A M}$, is added to the position of the pulse related to 16PPM modulation, which is located at $a_{16 P P M}$.

Looking at Fig. 2, and being $M_{P A M}$ the order of PAM modulation, the corresponding synchronization pulse becomes:

$$
s_{M_{P A M}}(t)=A_{0} \Pi\left(\frac{t-a_{16 P P M}-a_{P A M}}{b_{P A M}}\right)
$$

where, pulse width is:

$$
\begin{aligned}
& b_{P A M}=b_{P P M}=4 \tau \\
& a_{P A M}=4 \tau+\log _{2}\left(M_{P A M}\right) T_{g}+\left(\log _{2}\left(M_{P A M}\right)-1\right) T_{s}
\end{aligned}
$$




\begin{tabular}{|c|c|c|c|c|}
\hline PREAMBLE & $\begin{array}{c}\text { SYNCHRO } \\
\text { BREAKING }\end{array}$ & $\begin{array}{c}\text { STARTING } \\
\text { DELIMITER }\end{array}$ & DATA & $\begin{array}{c}\text { ENDING } \\
\text { DELIMITER }\end{array}$ \\
\hline
\end{tabular}

Fig. 3. Uplink communication: data frame.

From eq. (5), the delay associated to 16 PPM is

$$
a_{16 P P M}=6 \tau+\log _{2}(16) T_{g}+\left(\log _{2}(16)-1\right) T_{s}=26 \tau
$$

The term $4 \tau$ in eq. (8) properly locates pulse associated to 2PAM, at the end of the slot reserved to 16PPM, but maintaining a further guard time. So, the final equation for the pulse which identifies the order of PAM modulation, is:

$$
s_{M_{P A M}}(t)=A_{0} \Pi\left(\frac{t-26 \tau-a_{P A M}}{b_{P A M}}\right)
$$

Once the synchronization signal $y_{s}(t)$ affected by noise is received, and the first sample detected, the correct modulation format indicator is recovered according to the maximum likelihood (ML) criterion. Let us indicate as $s_{m}(t), m \in \mathfrak{M}$ the set of synchronization trails related to both PAM and PPM spanning the modulation orders we considered, we can individuate the kind of modulation and order on the basis of $\mu$ defined as

$$
\mu=\arg \max _{m \in \mathfrak{M}} \int_{T_{\text {start }}}^{T_{\text {end }}} s_{m}(t) y_{s}(t) d t .
$$

where $T_{\text {end }}$ is the time at which the information preamble ends. In our implementation, according to time reference in Fig. 2, $T_{\text {end }}=52 \tau$.

\section{B. Uplink Channel}

Once the correct modulation is set, user data are demodulated until a known end sequence is detected and the receiver waits for a new starting pulse. A silent time, in which no signal is transmitted, is provided between two consecutive frames in order to allow the receiver to evaluate the whole noise and measure SNR for choosing the next modulation to adopt. The main receiver communicates, through a control message via serial port, with the uplink transmitter, which modulates the control message and transmits the corresponding optical signal to the uplink receiver.

Each control message is repeated five times in the uplink frame. If these messages do not coincide, or an invalid word is detected, data transmission continues with the same modulation used in the previous frame. A low data rate OOK modulation, with Manchester encoding, ${ }^{1}$ is used in the uplink channel. The frame used for feedback messages is described in Fig. 3.

In particular, the frame is composed of:

- A preamble (1010101), useful for identifying an average value of the signal, in order to compute a threshold for distinguish high and low levels and decodify data (1 B).

- A synchronization breaking (1011101), which stops the preamble and it is used for hooking the encoding phase, in order to achieve a proper time recovery.

\footnotetext{
${ }^{1}$ When we refer to encode we aim at describing a source coding, that is, the association between bit streams and messages.
}

TABLE I

Uplink COMmunication: CONTROL MeSSAGES AND RELATED SNR RANGES

\begin{tabular}{|c|c|c|}
\hline Modulation & $\begin{array}{l}\text { Binary } \\
\text { Message }\end{array}$ & $\begin{array}{l}\text { SNR } \\
\text { Range } \\
{[\mathrm{dB}]}\end{array}$ \\
\hline 8 PAM & 00100110 & $>10$ \\
\hline 4 PAM & 01111001 & $8.3-10$ \\
\hline 2 PAM & 00000011 & $6-8.3$ \\
\hline 2 PPM & 00111110 & $4.7-6$ \\
\hline 4 PPM & 01001101 & $4-4.7$ \\
\hline 8 PPM & 00110011 & $3-4$ \\
\hline 16 PPM & 01000001 & $<1.7$ \\
\hline
\end{tabular}

- A starting delimiter (00000011) introduces user data.

- User data, containing the control message, namely a 8 b word associated to the next modulation that downlink transmitter will use for codify next frame. This word is repeated 5 times in the data field.

- An ending delimiter (00000100) after the user data, for indicating the end of the control message.

Each possible modulation is associated to the corresponding binary sequence, as outlined in Table I.

Control words have been chosen so as to guarantee a sufficient Hamming distance between them, in order to avoid that an error could transform a valid message to another valid message. A fundamental aspect regards the thresholds, in terms of SNR, in which the system changes modulation. Values reported in Table I have been chosen, after a preliminary experimental campaign, in order to avoid too many changes when environmental conditions are favourable, but at the same time, allow a high flexibility of the adaptive system in highly noisy environments.

Regarding the channel we do not provide, at this stage, an analytical model like for example line-of-sight based, diffusive non-light of sight since, as it will be clearer in the following, we resort to experimental data that, basing on the received signal, already contains all the channel and interference features.

\section{Signal Detection}

The first step of the detection procedure is to synchronize the symbols transmitted by the LED(s) in order to provide timing and, more, allowing the acquisition of other side information. This is used to understand the quality of the channel so as to take decision about the modulation scheme to be used for the target error rate and, then, communicating back this information to the transmitter.

\section{A. SNR and Channel Estimation Towards Modulation Control}

When only ambient noise is captured, we are able to measure its variance, that is, its power. This can be done by considering the samples gathered at the receiver when no transmission is active in the following way:

$$
\sigma_{y}^{2}=\frac{1}{N} \sum_{k=1}^{N}|y(k)|^{2} .
$$

In fact, when no transmission is active, we have that the power of the received signal $\sigma_{y}^{2}$ equates the power of the whole noise

$$
\sigma_{y}^{2}=\sigma_{w}^{2}
$$


where $\sigma_{w}^{2}=\sigma_{n}^{2}+\sigma_{I}^{2}$ accounts both for thermal $\left(\sigma_{n}^{2}\right)$ and ambient $\left(\sigma_{I}^{2}\right)$ noise. On the other hand, when transmission takes place, under the hypothesis of statistical independence among noise and signals sent by the transmitter, we have

$$
\sigma_{y}^{2}=|h|^{2} P_{x}+\sigma_{w}^{2} .
$$

$h$ being the channel gain.

SNR estimation, given by $|h|^{2} P_{x} / \sigma_{w}^{2}$, can be carried out by considering the following relationship

$$
\tilde{\beta}=\frac{\sigma_{y}^{2}-\sigma_{w}^{2}}{\sigma_{w}^{2}}=\frac{\sigma_{y}^{2}}{\sigma_{w}^{2}}-1
$$

and, once acquired this measure, it is possible to proceed to choose the modulation scheme that is expected to match the reliability constraints with the maximum allowed rate among those meeting the BER requirement that we map through SNR.

It is important to highlight now that, in case of interference mitigation procedures, it is possible to obtain lower values of $\sigma_{w}^{2}$ due to interference processing so as to increase the real (and not only the estimated) SNR so allowing to use higher modulation order without increasing the error rate of the system. From this point of view, the opportunity of hearing interference without any information signal is fundamental to acquire information about the disturbing elements. However, the simple measure of interference level, that is power, is not sufficient to provide statistical elements for interference mitigation [12]. In order to do so, we resort to linear prediction for the interference term so as to have the auto-correlation properties. In this regard, we have that the predicted interference (ambient noise) is

$$
\tilde{\eta}=\sum_{k=1}^{p} a_{k} \eta[n-k]
$$

$p$ being the order of the predictor and $a_{k}$ the coefficients that can be found by considering the Yule-Walker equations

$$
\sum_{k=1}^{p} a_{k} r_{\eta}[j-k]=r_{\eta}[j]
$$

In fact, the goal of the Yule-Walker equations is the minimization of mean square error defined as

$$
\mathcal{E}_{p}=E\left[|\eta-\tilde{\eta}|^{2}\right]
$$

with respect to the coefficients $a_{k}$ with $E($.$) representing the$ expected value operator. Hence the Levinson-Durbin algorithm can fast lead recursively to the solution as detailed in [13, Chapt.10]. The interference predicted is then subtracted to the received signal so as to try to clean it. In the above relationship, we have the term $r_{\eta}[j]$ that refers to the auto-correlation of the noise. The above term is characterized by both thermal noise (negligible) and ambient noise. Correlation can be obtained via very simple estimation procedure, that is, during a silenced phase, before training, that assumes that no transmission takes place so the only samples are given by noise. This allows to write

$$
r_{\eta}[j]=\frac{1}{N} \sum_{\ell=1}^{N} \eta[\ell] \eta[\ell+j]
$$

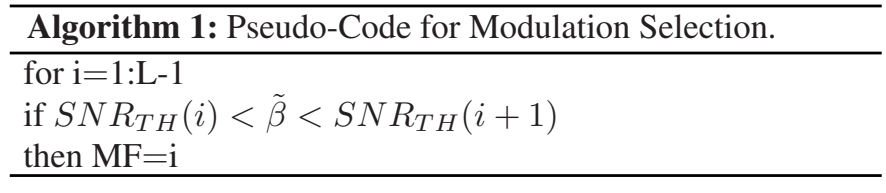

Hence the value of SNR after noise mitigation $\beta_{\mathcal{M}}$ is then given by

$$
\beta_{\mathcal{M}}=\frac{|h|^{2} P_{x}}{\sigma_{\epsilon}^{2}}
$$

$\sigma_{\epsilon}^{2}$ being the residual noise after mitigation that is lower than $\sigma_{w}^{2}$ since it is given by $\sigma_{\epsilon}^{2}=\sigma_{n}^{2}+\mathcal{E}_{p}$, where $\mathcal{E}_{p}$ is the already mentioned estimation mean square error minimized according to eq. (18).

In this direction, we equip the receiver with a table where we have in each row, SNR required for the target error rate for each modulation scheme. Hence, it is able to measure periodically the SNR by introducing a silenced phase during which noise samples are captured and a training one so as to re-estimate both channel and SNR. Too much frequent re-estimation leads to waste transmission resources (no information data sent) while too sporadic signalling leads to having outdated information. This can be analytically described as in the pseudo-code reported in Algorithm 1.

Thus meaning that we span all the L different modulation formats by checking if the SNR measured is in the range limited by two SNR thresholds for the target error rate $\left[S N R_{T H}(i), S N R_{T H}(i+1)\right]$ so as to choose as modulation format the i-th one.

\section{B. Channel Estimation Cadence}

When dealing both with a modulation selection and interference mitigation procedure it is reasonable to assume that channel does not change if the propagation environment does not. Changes may occur when the propagation environment is affected by more reflections (object around disappear or appear) and/or relative distance between transmitter and receiver changes. Besides, light sources may induce interference if the illumination is not uniform thus becoming position dependent. From this aspect, it is possible to understand the need of considering how much frequent the estimation procedure, as well as, interference acquisition must take place. The idea to allow reliable channel estimation, without wasting time to try to estimate channel when it is not needed is to check, during time, for possible changes. This should be done both for channel and interference. First of all let us focus on the estimation of channel. We may resort to a criterion, like for example minimum mean square error, from the literature as reported in [13, Chapt.5] by using training sequences. The training is the already described signalling scheme depicted in Fig. 2. However, even though we use training symbols, we can use the SNR estimation in eq. (20) since it is possible to infer that since $h$ is always positive and real, it is given by

$$
\tilde{h}=\sqrt{\frac{\beta_{\mathcal{M}} \sigma_{n}^{2}}{P_{x}}}=\sqrt{\frac{|h|^{2} \sigma_{n}^{2}}{\sigma_{n}^{2}+\mathcal{E}_{p}}}
$$




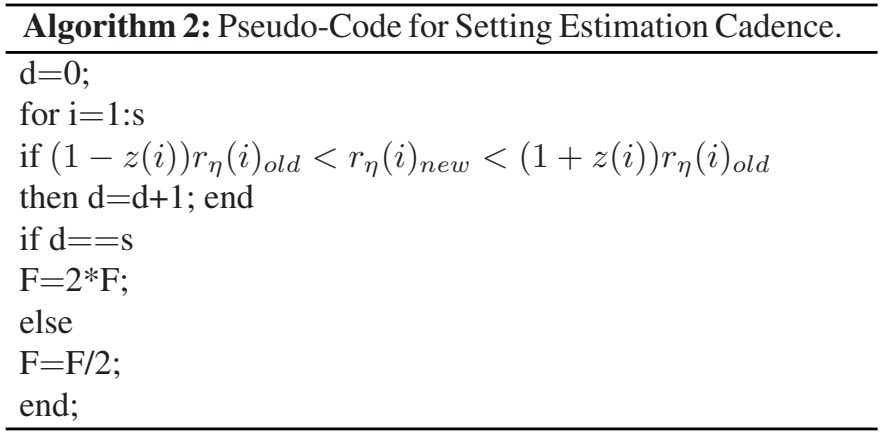

hence a good interference mitigation leads to reliable channel estimation since $\mathcal{E}_{p}$ is very low and channel estimation converges toward the real value.

Secondly, let us consider on the rate reduction induced by the acquisition of the statistics. If we assume that the procedure for interference acquisition last $A T_{p}$ seconds and the channel estimation/synchronization involves $16 T_{p}$, if it happens every $F$ data symbols, we have the rate scales in the following way:

$$
\mathcal{G}=R\left(1-\frac{16+A}{F+16+A}\right)
$$

where $R$ is the rate of the transmission while $\mathcal{G}$ is the net rate. In this regard acquiring both the statistics of noise and channel is fundamental and a proper threshold should be set in order to have the opportunity to judge the channel as changed or not. The use of a threshold is a reasonable way since asking that the channel (or the noise) must be exactly the same (identity) is a nonsense for our purpose since minimal changes may not have any effect on performance. Thus, we propose the mechanism we detail in Algorithm 2. It is possible to appreciate that we focus on the first $s$ terms of the noise correlation and if nothing changed considerably (we check the fluctuation through $z(i)$ ) then the noise is considered as invariant and we can send more data $(\mathrm{F}$ $=2 \mathrm{~F}$ ) without re-estimating the noise, otherwise we need to re-estimate it.

An analogue procedure must be performed on the basis of the SNR fluctuations (using the received power in place of the correlation) to increase or decrease $F$.

\section{Digital Demodulation}

About digital demodulation we resort to the maximum likelihood (ML) criterion starting from the sequence of the received signal after noise removal. In this regard we can refer to the received and de-noised signal sequence as

$$
z[n]=y[n]-\tilde{\eta}[n]
$$

Basing of the modulation format, PAM or PPM, we proceed to consider also the effect of channel so as to resort to the following metric that is valid for PPM

$$
v_{\ell}=z^{T} \tilde{h} s_{\ell}
$$

$\tilde{h}$ being the estimated channel, $s_{\ell}$ the vector shape of the PPM transmitted signal associated to the $\ell$-th symbol. The metric $\mathbf{v}_{\ell}$ is then used for digitally demodulating the received signal

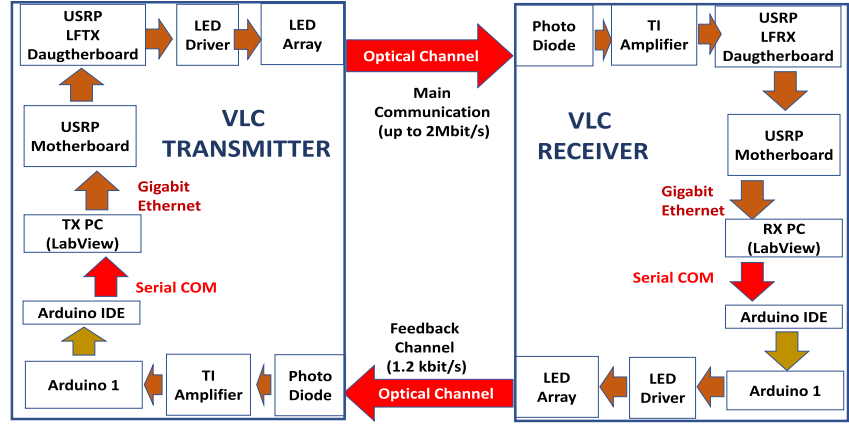

Fig. 4. VLC bidirectional system: main hardware components.

according to the ML criterion as

$$
\hat{l}=\underset{\ell=\{0, \ldots M-1\}}{\operatorname{argmax}} \boldsymbol{v}_{\ell}
$$

For what concerns PAM, still starting from 23, we have that the decision criterion ML leads to the following detected symbol

$$
\hat{l}=\underset{\ell=\{0, \ldots M-1\}}{\operatorname{argmin}}\left\|\mathbf{z}-\tilde{h} \mathbf{s}_{\ell}\right\|^{2}
$$

\section{SySTEM IMPLEMENTATION}

Till now we described the system we want to develop by resorting to analytical description both of signals at the transmitter and the procedure for mitigating and counterbalance the different impairments coming from the transmission/reception itself. In line with other works from the literature that present the analytical content of the proposal (see [14], [15]), we detail now how the system we described in the previous sections has been implemented in a real fully-functioning two-way optical wireless communication link.

At this stage, we want to emphasize that the choice of modulation scheme is in charge of the receiver that, via the uplink channel, must communicate back the word corresponding to the modulation scheme. For this motivation, the whole architecture of the system has been designed in order to achieve an effective coordination between receiver and transmitter and a robust deliverance of feedback message.

\section{A. Hardware Components}

A simplified scheme of the hardware elements composing our bidirectional VLC system is depicted in Fig. 4.

The main optical communication between the transmitter and the receiver is performed using two Universal Software Radio Peripheral (USRP) 2922, a universal platform for software defined prototyping, provided by National Instruments. In order to allow the platforms to modulate and demodulate the signal in the range [0-30 MHz] the original front-end has been substituted by a low frequency receiving daughter-board, both provided by Ettus.

However, the original motherboards have been maintained in the system. The connection between the USRP and the the host PCs are guaranteed by a Gigabit Ethernet cable. The communication between motherboards and daughter-boards and between 


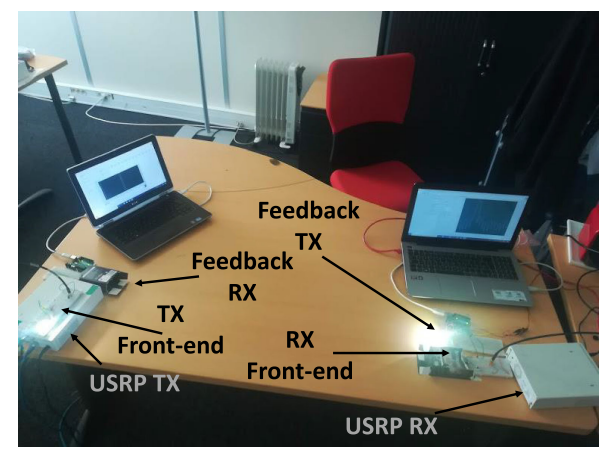

Fig. 5. VLC bidirectional system: hardware components.

the daughter-boards and the front-ends are performed by SMA connections. A picture of the corresponding setup is provided in Fig. 5.

Since the signal coming out from the transmitting USRP is in the range $[-1 \mathrm{~V}, 1 \mathrm{~V}]$, a proper bias tee and a single stage amplifier are used for allowing Led driving in the proper range $[0 \mathrm{~V}-5 \mathrm{~V}]$. A $12 \mathrm{~V}$ power supply is used for feeding the LED array. In order to allow the receiving signal to be correctly detected in the dynamic range of the receiving USRP, ([-0.9 V-0.9 V]), an amplifying and conditioning network has been designed. The first amplifier network allows the photodiode to be correctly polarized and the signal to be in the correct range for the second amplifying network, which performs the high gain trans-impedance amplification. An additive DC block capacitor mitigates the high DC component due to amplifiers, in order to avoid saturation of the USRP receiver. A $12 \mathrm{~V}$ power supply feeds both the amplifiers. In the uplink communication, the optical part of the circuit (LEDs and photodiodes) is identical to the one in the main communication, but analog to digital conversion and digital signal processing are performed using Arduino Uno. Since the dynamic allowed by Arduino card ([0 V-5 V ]) is larger than the one of USRP, bias tee and amplification are not needed in the feedback transmitting stage. The uplink receiving front-end presents the same components of the main receiver front-end, but the value of circuit components changes accordingly to the different dynamic. In both receiving and transmitting stages, a serial connection (allowing a standard $115200 \mathrm{Bd}$ rate) is used for communicate between the Arduino and the corresponding PC. In order to avoid a significant cross-talk between the main communication and the feedback communication, a paper box with black inner walls has been placed around the receivers. Since uplink channel does not need a very high data rate, a low frequency OOK (up to some $\mathrm{kHz}$ ) with Manchester encoding (eliminating the problem of DC components) has been used. Since PPM communication works at much higher blinking frequencies (up to some $\mathrm{MHz}$ ), the interference between the main communication and the feedback communication is negligible. Feedback receiver circuit and main VLC transmitter shares a $12 \mathrm{~V}$ feeding power supply, while the power supply of the main receiver is also used for feeding uplink transmitting LED. In both cases, some shunt capacitance have been used in order to reduce, as far as possible, high frequency noise components due to the feeding network.

\section{B. Software Defined Operations}

According to software defined paradigm, most of signal processing operation (i.e. signal generation, filtering, modulation, demodulation, time recovering and data evaluation) are performed via software in order to reduce hardware costs and improve system flexibility. The main high data rate communication, involving the two USRP platforms, is completely managed using LabView, a system-design platform and development environment for a visual programming language from National Instruments. Furthermore, proper LabView subroutines have been designed for data elaboration, real time performance evaluation, generation of control messages and the coordination of adaptive operation. Uplink communication is completely managed using the Arduino integrated development environment (IDE), a cross-platform application written in the programming language Java. Also in this case a software defined approach has been employed. The code is directly flashed in Atmel 8-bit AVR micro-controller, while standard IDE commands for read and write operations have been replaced with appropriate low level instruction in order to speed up the access to the registers, and consequently, improve data rate communication of uplink stage. Main communication and uplink communication works at different data rate, so, in order to preserve real time operations and avoid to waste system performance, subroutines in LabView and the code flashed in the Arduino are executed in parallel. The exchange of control messages between LabView and Arduino takes place through serial communication. In order to preserve a multi-thread logic, a concurrent dedicated subroutine is provided in both transmitting and receiving stages for managing serial communication without stopping signal processing. Once performance related to a portion of received signal are evaluated, a new control message is immediately generated via LabView and transmitted to the serial port. An Arduino routine reads the stream incoming from the serial port, encodes and transmits feedback message. On the other side, the uplink RX Arduino receives and demodulates the feedback message. Thereafter, the control message is transmitted to the serial port, and a parallel subroutine, executed in LabView, continuously listens for new messages and periodically communicates with the main subroutine. If a message is lost, or if it is corrupted, the main communication is performed using previous parameters and there is no lack of communication even if the feedback mechanism fails. An identification preamble guarantees the main transmitter and receiver to be set with the same communication parameters (i.e. type and order of modulation). A single pulse before the identification preamble is used for starting the communication (unsqueltch) and acting as a unique synchronization message for all the modulation types allowed by the system. Details about these operations are outlined in Algorithms 3, 4, 5 and 6, while more information about signals and data involved in the process are outlined in next subsection.

\section{Numerical Results AND Tests}

In this Section we detail the setup both for computer simulation and tests by showing the main characteristics of the 

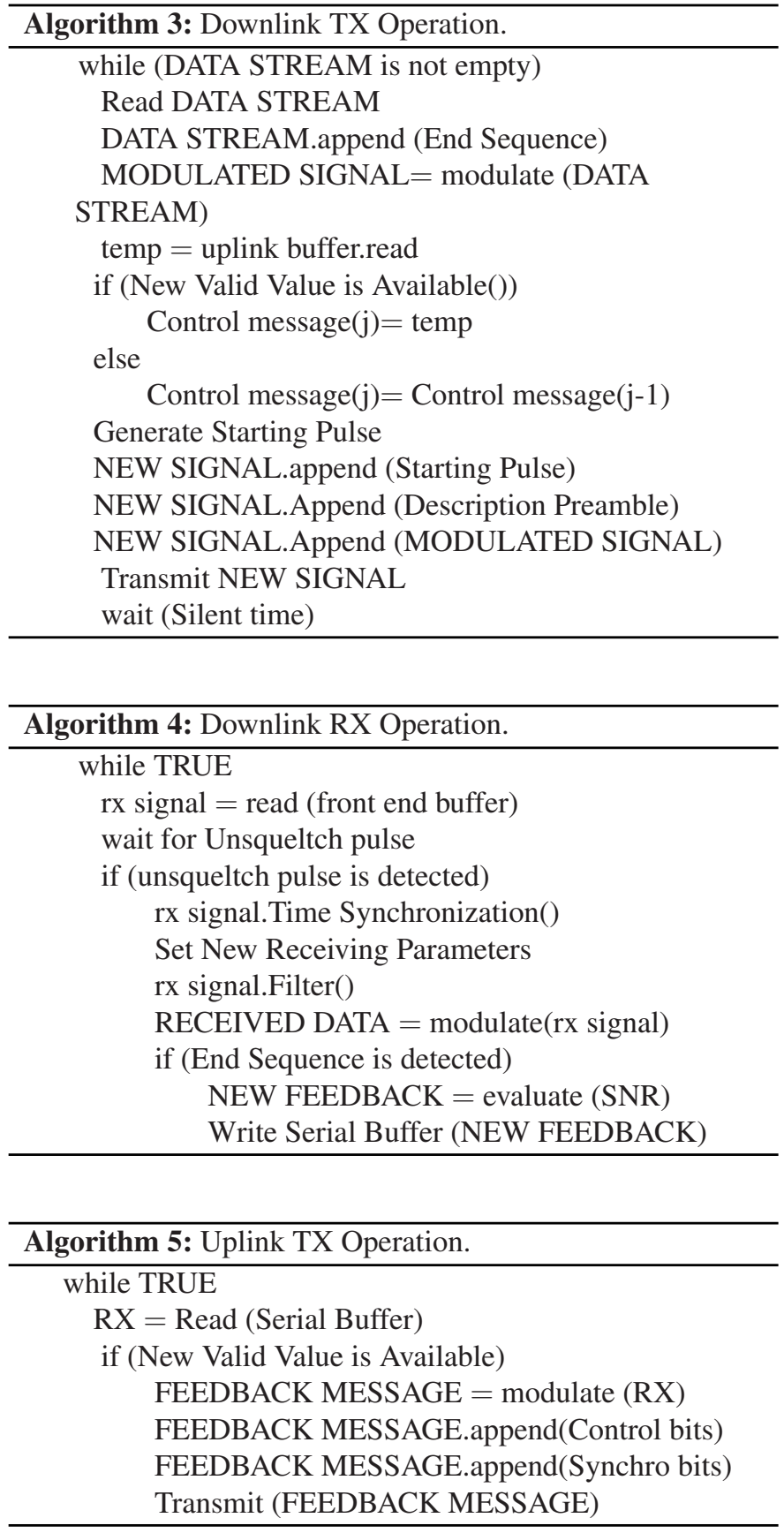

transmitter and receiver. A short recap of the main parameters can be found in Table II.

The use of a pre-amplifying network allows to properly bias the trans-impedance stage. Since a high trans-impedance gain can be achieved only using a high value resistance in the path, a significant amount of additive noise is added in the circuit and a proper feedback capacity should be added for avoiding instability. However, main other parameters are fixed and depend of the correct choice of lamps and photo-detectors. LED lamps have been chosen to have an adequate trade off between cost, brightness and electrical power needed for feeding. A photodiode with an adequate responsivity in the same optical frequency range of LED maximum emission has been selected.
TABLE II

EXPERIMENTAL VALIDATIONS: MAIN PARAMETERS OF TRANSMITTING AND RECEIVING HARDWARE STAGES

\begin{tabular}{|c|c|c|c|}
\hline \multicolumn{2}{|c|}{ Receiver } & \multicolumn{2}{|c|}{ Transmitter } \\
\hline $\begin{array}{c}\text { Photodiode } \\
\text { Model }\end{array}$ & $\begin{array}{c}\text { CENTRONIC } \\
\text { OSD15-5T }\end{array}$ & $\begin{array}{l}\text { LED } \\
\text { Model }\end{array}$ & $\begin{array}{c}\text { CREE } \\
\text { MKRAWT } \\
-02-0000 \\
-0 \text { D00J2051 }\end{array}$ \\
\hline Active Area & $15 \mathrm{~mm}^{2}$ & Maximum Power & $15 \mathrm{~W}$ \\
\hline $\begin{array}{l}\text { Responsivity } \\
\quad(436 \mathrm{~nm})\end{array}$ & $0.18-0.21$ & $\begin{array}{c}\text { Effective } \\
\text { Power }\end{array}$ & $4 \mathrm{~W}$ \\
\hline Dark Current & $1-5 \mathrm{nA}$ & $\begin{array}{l}\text { Maximum } \\
\text { Light Flux }\end{array}$ & $1040 \mathrm{~lm}$ \\
\hline Field of View & $45 \mathrm{deg}$ & Field of View & $120 \mathrm{deg}$ \\
\hline $\begin{array}{c}\text { Noise } \\
\text { Equivalent } \\
\text { Power } \\
(436 \mathrm{~nm})\end{array}$ & $5.5 * 10^{-14}$ & Forward Voltage & $12 \mathrm{~V}$ \\
\hline Capacitance & $80 \mathrm{pF}$ & $\begin{array}{c}\text { Maximum } \\
\text { Forward Current }\end{array}$ & $1200 \mathrm{~mA}$ \\
\hline Bandwidth & $29.1 \mathrm{MHz}$ & $\begin{array}{c}\text { Maximum } \\
\text { Switching Speed }\end{array}$ & $35 \mathrm{MHz}$ \\
\hline $\begin{array}{c}\text { Shunt } \\
\text { Resistance }\end{array}$ & $200 M \Omega$ & Reverse Voltage & $5 \mathrm{~V}$ \\
\hline Rise Time & $12 \mathrm{~ns}$ & Number of LED & 5 \\
\hline $\begin{array}{c}\text { Trans } \\
\text { Impedance } \\
\text { Amplifier Gain }\end{array}$ & $1 \mathrm{M} \Omega$ & $\begin{array}{c}\text { Optical } \\
\text { Concentrator } \\
\text { Gain }(6 \mathrm{~m})\end{array}$ & 5 \\
\hline
\end{tabular}

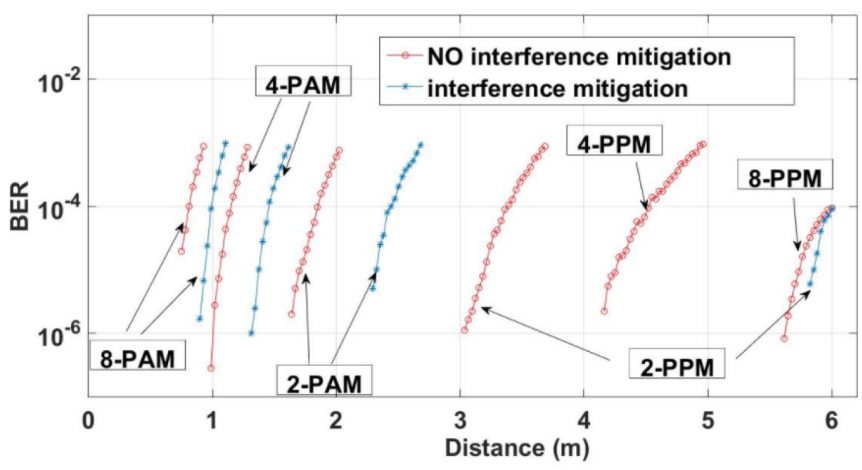

Fig. 6. BER of adaptive scheme with and without interference mitigation when uniform lighting is used.

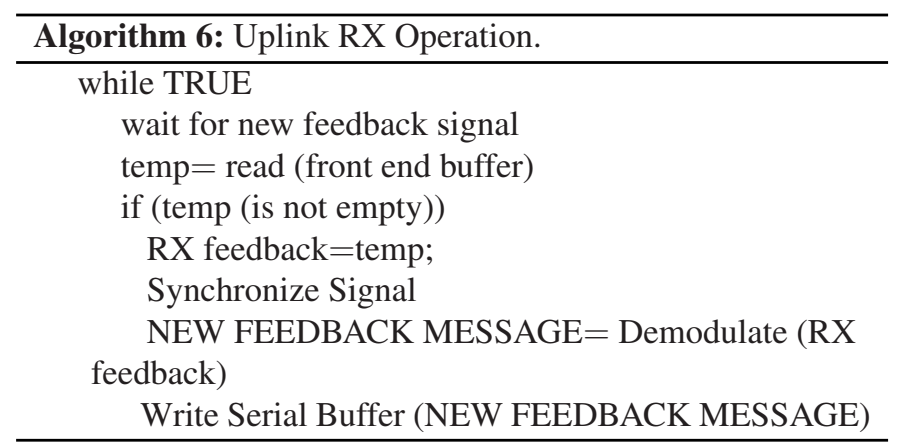

Furthermore, a trade off between costs, available bandwidth and low parasitic effect has been considered.

In Fig. 6 we show the performance of two different schemes. The first one is related to the absence of interference mitigation procedure and, by moving slowly $1 \mathrm{~m} / \mathrm{s}$ the receiver far from the transmitter, we increase distance with a uniform 


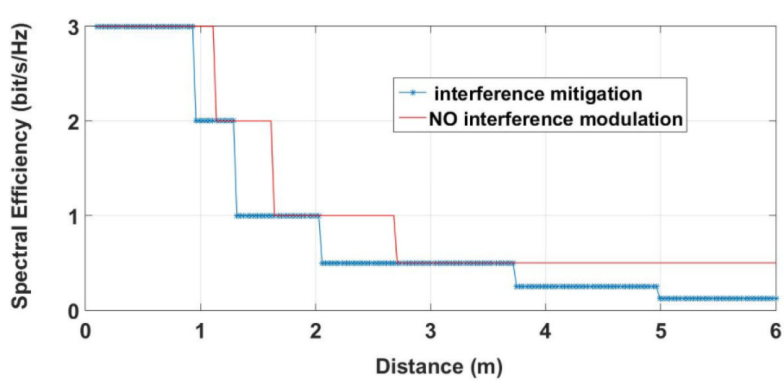

Fig. 7. Spectral Efficiency of adaptive scheme with and without interference mitigation when uniform lighting is used.

interference level. The second scheme is related to the same situation where interference mitigation is acted according to the above explanation.

It is possible to note that by setting the BER threshold to $10^{-3}$, we have an erratic behavior of the performance in the no interference mitigation case, since when the SNR is approaching the reference value stored in the table, a modulation switch is acted and its new performance can be, as in this case, sufficiently lower. Hence, this erratic behaviour continues by switching from 8-PAM till to the less complex 2-PAM and then from 2-PPM to 8-PPM. By recalling that spectral efficiency for PAM is $\log _{2 M}$ and for PPM is $M^{-1} \log _{2 M}$, the values related to the modulation range from 8-PAM to 8-PPM, reduces the spectral efficiency from $3 \mathrm{~b} / \mathrm{s} / \mathrm{Hz}$ (8PAM) to $0.375 \mathrm{~b} / \mathrm{s} / \mathrm{hz}$ (8PPM). For the reported scenario it is possible to show how the spectral efficiency scales by considering the behavior of the two curves in Fig. 7 .

It is important to note that sometimes the curve seems to disappear. This is due to the fact that the error rate is lower than $10^{-7}$ and no errors incur in our tests.

The performance exhibited by the interference mitigation approach are really similar in the shape with respect to the no-mitigation even though the BER we achieve is lower and also the switch is operated at a higher distance, so allowing, at the same distance, a higher rate or, for the same rate, higher distance.

As previously mentioned, the cadence we use for estimating both channel and interference is really important since we can be outdated and not able to react to channel and/or external interference changes. In Fig. 8 we show exactly the same test performed in Fig. 6 with interference mitigation with the difference of considering also not adaptive mechanism to acquire information about interference and channel. In particular the curve labeled as fixed update is related to sending pilots and acquire interference every $10^{6}$ symbols and when the tracking is performed according to the previously detailed mechanism.

In Fig. 9 it is possible to appreciate the behaviour with respect to $F$ and $A$ of the net rate. When $A$ increases the rate decreases while increasing the number of consecutive data symbols increase the net rate at the expenses, as it will be appear clearer in the following, of the reliability. About the price to be paid to be updated about interference and channel, it is possible to appreciate that if the estimation cadence is too slow, we are unable to be updated so the real SNR is different from the estimated (supposed) one so we change modulation

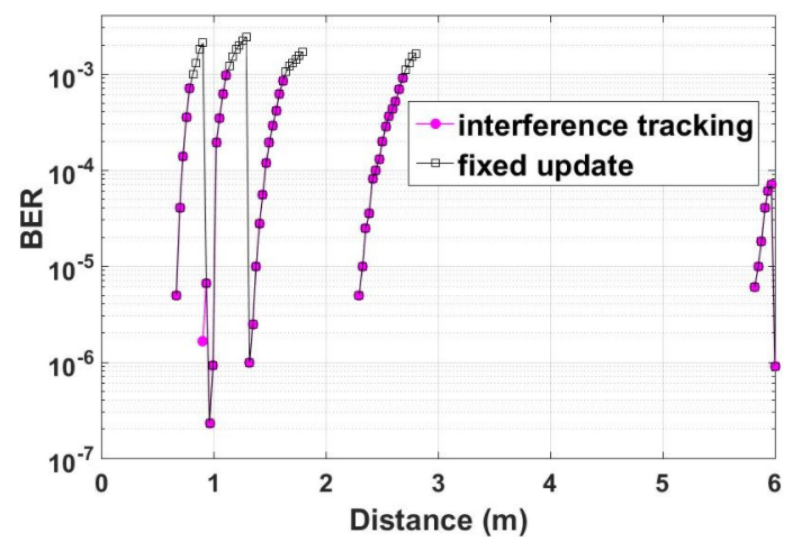

Fig. 8. BER of adaptive scheme with and without interference mitigation when uniform lighting is used and different channel updates are performed.

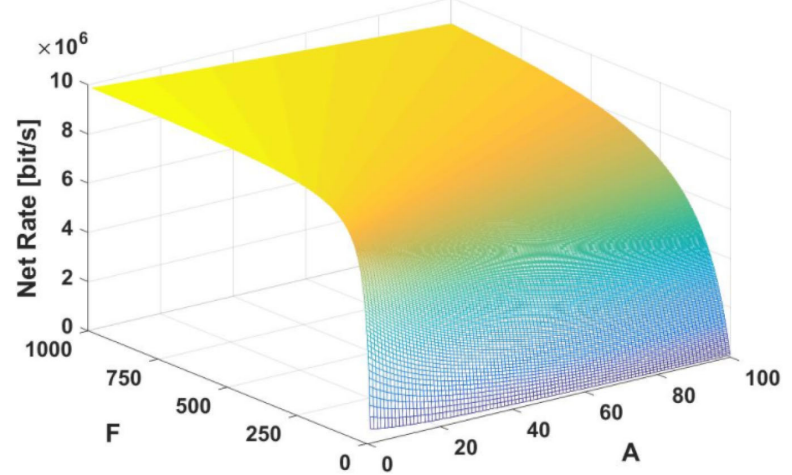

Fig. 9. Net rate as a function of noise acquisition length $A$ and estimation cadence $F$

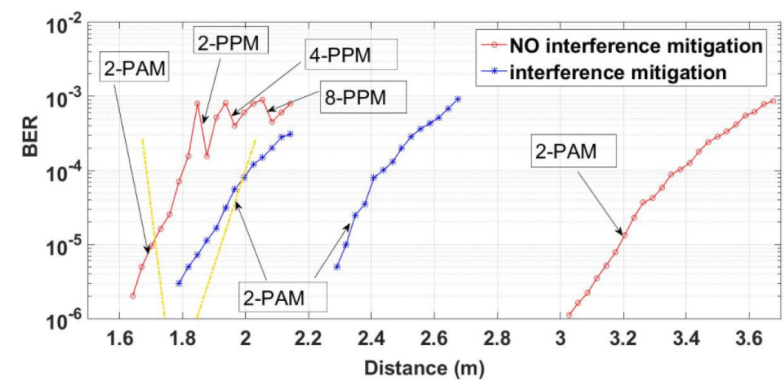

Fig. 10. BER of adaptive scheme with and without interference mitigation when non-uniform lighting is used. The yellow lines describe the propagation of disturbing light source over the distance.

scheme late with respect to the distance where the receiver is based so violating the constraint on BER required by the system performance. Increasing the speed of the receiver may highlight this issue since the channel changes faster.

Furthermore, in order to test the performance of the interference mitigation, we remove the hypothesis of uniform distributed disturbing light and pose one light source at 1.87 meters from the transmitter. In Fig. 10 it is possible to see the position of the lighting source represented by the yellow box, and the yellow lines describe the propagation over the distance. It is important to highlight that while the case of no interference 
suppression, due to the additional interference changes fast the modulation scheme scaling from 2-PAM till to 8-PPM, the interference mitigation case do not change since 2-PAM is still able to guarantee the target BER, thus meaning that the SNR after mitigation is still above the threshold that states the switch between 2-PAM and 2-PPM.

\section{CONCLUSION}

Visible Light Communication is gaining more and more large interest as cost-effective and energy efficient communication wireless technology. In order to be able to efficiently exploit the high potential and ubiquitous character of VLC it is of paramount importance to develop bi-directional systems, in order to fully exploit the information state at the receiver for adapting the system to the high dynamic environmental conditions. In this work we have designed and implemented a complete bi-directional system based on USRP and commercial LED and photodiode. Our main objective was to enable the receiver to send back on a dedicated back channel information regarding a smart selection of the most suitable modulation technique. This selection is based on SNR and threshold values (in terms of BER and data rate) defined on the basis of the application. The system needs to correctly synchronize the downlink and uplink channel, avoiding mutual interference. We have implemented the logic selection at the receiver and demonstrate the effectiveness of the adaptive system when adding interference to the communication system.

\section{REFERENCES}

[1] H. Burchardt, N. Serafimovski, D. Tsonev, S. Videv, H. Haas, "VLC: Beyond point-to-point communication," IEEE Commun. Mag., vol. 52, no. 7,pp. 98-105, Jul. 2014.

[2] Q. Wang, D. Giustiniano, D. Puccinelli, OpenVLC: " Software-defined visible light embedded networks," in Proc. 20th Annu. Int. Conf. Mob. Comput. Netw. (ACM MobiCom 2014), Maui, Hawaii, USA, 2014, pp. 15-20.

[3] C. Wang, H. Y. Yu, Y. J. Zhu, "A long distance underwater visible light communication system with single photon avalanche diode," IEEE Photon. J., vol. 8, no. 5, pp. 1-11, Oct. 2016.

[4] Y. Zhuang et al., "A survey of positioning systems using visible LED lights," IEEE Commun. Surv. Tut., vol. 20, no. 3, pp. 1963-1988, Oct./Nov. 2018,

[5] A. Costanzo, V. Loscri, "Error compensation in indoor positioning systems based on software defined visible light communication," Phys. Commun., vol. 34, pp. 235-245, 2019.
[6] Y. H. Kim, W. A. Cahyadi, Y. H. Chung, "Experimental demonstration of VLC-Based vehicle-to-vehicle communications under fog conditions," IEEE Photon. J., vol. 7, no. 6, pp. 1-9, Dec. 2015.

[7] E. A. Lee and D. G. Messerschmitt, Digital Communication, 2nd ed., Norwell, MA, USA: Kluwer, 1994.

[8] D. J. F. Barros, S. Wilson, J. M. Kahn, "Comparison of orthogonal frequency-division multiplexing and pulse-amplitude modulation in indoor optical wireless links," IEEE Trans. Commun., vol. 60, no.1, pp. 153-163, Jan. 2012.

[9] Z. Wang, C. Yu, W. D. Zhong, J. Chen, W. Chen, "Performance of a novel LED lamp arrangement to reduce SNR fluctuation for multi-user visible light communication systems," Opt. Exp., vol. 20, pp. 4564-4573, 2012.

[10] P. Miao, L. Wu, Z. Chen, "Anti-noise modem for visible light communication systems using the improved m-ary position phase shift keying," $A E U$ - Int. J. Electron. Commun., vol. 85, pp. 126-133, 2018.

[11] A. Costanzo and V. Loscri, "Demo: A context aware algorithm for an adaptive visible light communication system," in Proc. EWSN 2018-Int. Conf. Embedded Wireless Syst. Netw., Feb. 2018.

[12] A. Costanzo, V. Loscri, M. Biagi, "A noise mitigation approach for VLC systems," in Proc. 2019 Global LIFI Congr. (GLC), Paris, France, Jun. 2019, pp. 69-74.

[13] J. G. Proakis, M. Salehi, Digital Communication, 5th ed., Hoboken, NJ, USA: Wiley, 2008.

[14] D. Tsonev, S. Videv, H. Haas, " Unlocking spectral efficiency in intensity modulation and direct detection systems," IEEE J. Sel. Areas Commun., vol. 33, no. 9, pp. 1758-1770, Sep. 2015.

[15] A. A. Al-Hameed, S. H. Younus, A. T. Hussein, M. T. Alresheed, J. Elmirghani, " LiDAL: Light detection and localization," IEEE Access, vol. 7, pp. 85645-85687, 2019.

[16] Q. Wang, D. Giustiniano, O. Gnawali, "Low-cost, flexible and open platform for visible light communication networks," Hotwireless '15. ACM, New York, NY, USA, pp. 31-35, 2015.

[17] A. Costanzo, V. Loscri, S. Costanzo," Adaptive dual color visible light communication (VLC) system," Trends Adv. Inform. Syst. Technol., vol. 2, pp. 1478-1487, 2016.

[18] A. Costanzo, V. Loscrí, V. Deniau, J. Rioult., “ On the interference immunity of visible light communication (VLC)," in Proc. IEEE Global Commun. Conf., 2020.

[19] C. G. Gavrincea, J. Baranda, and P. Henarejos, "Rapid prototyping of standard-compliant visible light communications system," IEEE Commun. Mag., vol. 52, no. 7, pp. 80-87, Jul. 2014.

[20] E. Knightly, Y. Qiao, H. Haas, "A software-defined visible light communications system with WARP", in Proc. 1st ACM Workshop Visible Light Commun. Syst., 2014, pp. 2434-2442.

[21] A. Galisteo, D. Juara, D. Giustiniano, "Research in visible light communication systems with openVLC1.3," IEEE 5th World Forum Internet Things, 2019, pp. 539-544, 2019.

[22] B. Aly, M. Elamassie, M. Uysal, E. Kinav., "Experimental evaluation of unipolar OFDM VLC system on software defined platform," in Proc. 15th Int. Conf. Telecommun., 2019, pp. 1-6. 